\title{
Activity recognition using one-versus-all strategy with relief-f and self-adaptive algorithm
}

\begin{abstract}
Many researchers dealing with smartphone sensors to recognize human activities using machine learning algorithms. In this paper, we proposed One-versus-All (OVA) strategy with relief-f and self-adaptive algorithm to recognize these activities. Relief-f used to rank the features and prune insignificant features, self-adaptive algorithm selects the relevant ones, and OVA transform features into a series of two-class classification problems, and later recognized by based classifier. Experiments were carried out to study the performance of our proposed algorithm using publicly activity datasets namely Physical Activity Monitoring for Aging People. It covers eighteen activities comprising various simple and complex activities. The performance of our method is compared to One-versus-One algorithm. The results have significantly promised an improvement of activity recognition level, mainly involving very similar activities.
\end{abstract}

Keyword: Human activity recognition; Relief-f and self-adaptive algorithm; One-versus-all; One-versus-one; Physical activity monitoring 